\title{
Electron correlation effects and ferromagnetism in Iron
}

\author{
Pablo Pou, Fernando Flores, José Ortega, Rubén Pérez and Alfredo Levy Yeyati \\ Departamento de Física Teórica de la Materia Condensada, Universidad Autónoma de Madrid, E-28049 Madrid, Spain
}

(draft of November 20, 2018)

\begin{abstract}
Electron correlation effects in Fe are analyzed using a first principles LCAO-scheme. In our approach, we first use a local orbital DFT-LDA solution to introduce a Hubbard Hamiltonian without fitting parameters. In a second step, we introduce a many-body solution to this Hamiltonian using a DMF approximation. Our analysis shows that magnetism in Fe is an effect associated with the first atomic Hund's rule. Moreover, we also find important correlation effects in the Fe-spin polarized DOS. The photoemision spectra is explained using a value of $U^{\text {eff }}$ as large as $4 \mathrm{eV}$, provided the satellite peaks appearing around $3-5 \mathrm{eV}$ below the Fermi energy are interpreted appropriately.
\end{abstract}

PACS numbers: 71.10.-w, 75.50.Bb, 75.10.Lp, 71.15.Mb

The electronic properties of ferromagnetic metals are still a subject of controversy [1] 3]. Although DFTLDA calculations yield the correct magnetization for the itinerant-electron ferromagnets $\mathrm{Fe}, \mathrm{Co}$ and $\mathrm{Ni}$, the origin of ferromagnetism in these metals and the role of electron correlations are not completely well-understood (e.g. see [国); in particular, the relative importance of the local Coulomb interaction for $d$ orbitals, $U^{\text {eff }}$, versus intraatomic exchange (first Hund's rule) is not completely established. Even from an experimental point of view, there is a lack of agreement on the existence or not of a satellite peak in the photoemission spectrum of iron around $5 \mathrm{eV}$ below the Fermi energy [2].

In the conventional view of itinerant ferromagnetism [5], spin polarization is determined by the Stoner parameter, $I$, that defines the energy of the atomic $d$-orbitals as $\left(E_{d}-I n_{d}\right), n_{d}$ being the occupation number of the orbital under consideration. In the case of Fe, DFTLDA calculations yield a value $I=3.9 \mathrm{eV}$ [6], and a surprisingly large value of $U^{\text {eff }} \sim 4-6 \mathrm{eV}, 0,8$ for the effective Coulomb interaction between the $d$-electrons. Since the atomic-like properties of the $d$-states are of crucial importance for the magnetic properties of these materials, LCAO methods provide the appropriate conceptual framework to understand those properties and to analyze the role of electron correlations. In LCAOtheories of ferromagnetism, including Hubbard Hamiltonians, $I$ is written as $\left(\widetilde{U}^{\text {eff }}+4 J^{x}\right)[9]$, where $J^{x}$ defines the screened intrasite exchange interaction between the atomic $d$-electrons having the same spin; it is commonly accepted that $J^{x}$ practically coincides with its atomic value [7]. In the case of $\mathrm{Fe}, J^{x}=0.83 \mathrm{eV}$ and, therefore, we should take $\widetilde{U}^{\text {eff }} \sim 0.6 \mathrm{eV}$ to recover the value $I=3.9$ $\mathrm{eV}$ that corresponds to the correct magnetization. This result suggests the presence of dramatic electron correlation effects in $\mathrm{Fe}$, which would be responsible for the renormalization of $U^{\text {eff }}$ from $\sim 5 \mathrm{eV}$ to $\widetilde{U}^{\text {eff }} \sim 0.6 \mathrm{eV}$. On the other hand, the value of $U^{\text {eff }}$ inferred from the photoemission spectra 10 12 by identifying photoemission peaks with quasi-particle peaks yields $U^{\text {eff }} \approx 2 \mathrm{eV}$ [1,8]. This result seems to indicate that electron correla- tion effects for Fe are not strong, in contradiction with the previous analysis.

The purpose of this paper is to show that these apparent contradictions disappear once electron correlation effects 113] are properly analyzed using a first-principles LCAO-scheme. In our approach, reminiscent of $\mathrm{LDA}+\mathrm{U}$ [14], we first formulate a Local Density solution for a generalized Hubbard Hamiltonian. This LD-solution provides the link between the generalized Hubbard Hamiltonian and local orbital DFT-LDA methods, allowing us to obtain that Hamiltonian from first principles, without having to introduce fitting parameters. In a second step, we introduce a many-body solution for the Hubbard Hamiltonian using a Dynamical Mean Field (DMF) approximation 15: in this way we analyze the spinpolarized electron density of states for $\mathrm{Fe}$ and compare it with the experimental evidence [10 12]. From our analysis, we obtain two different results. First, using our LD-solution for the Hubbard Hamiltonian, we show that electron correlation effects screen strongly the effective Coulomb interaction contributing to the Stoner parameter: in this sense, $\widetilde{U}^{\text {eff }}$ is not larger than $0.6-0.7 \mathrm{eV}$. We find, however, that the effective interaction appearing in the Hubbard Hamiltonian is around $4 \mathrm{eV}$, in reasonable agreement with other first-principles calculations [7,8]; using this value and the many-body techniques mentioned above, we also find that the spin-polarized DOS for Fe is in good agreement with the photoemission data, provided we interpret appropriately the satellite peaks appearing in the spectrum around 3-5 eV below the Fermi energy [8].

Our starting point is the generalized Hubbard Hamiltonian:

$$
\begin{aligned}
& \hat{H}=\hat{H}^{O E}+\frac{1}{2} \sum_{i, \alpha \sigma \neq \beta \sigma^{\prime}} U_{i} \hat{n}_{i \alpha \sigma} \hat{n}_{i \beta \sigma^{\prime}} \\
& -\frac{1}{2} \sum_{i, \alpha \sigma \neq \beta \sigma} J_{i}^{x} \hat{n}_{i \alpha \sigma} \hat{n}_{i \beta \sigma}+\frac{1}{2} \sum_{i \neq j}^{\alpha \sigma, \beta \sigma^{\prime}} J_{i \alpha, j \beta} \hat{n}_{i \alpha \sigma} \hat{n}_{j \beta \sigma^{\prime}} ;
\end{aligned}
$$

where $\hat{H}^{O E}$ defines a one-electron contribution, and $U_{i}$ and $J_{i \alpha, j \beta}$ the intrasite and intersite coulomb interac- 
tions between different orbitals $\phi_{i \alpha}$ and $\phi_{j \beta}$ (for the sake of simplicity, $U_{i}$ is an average of the different interactions inside the $i$-site); we also introduce the intrasite exchange coulomb interaction, $J_{i}^{x}$, associated with the first atomic Hund's rule. Eqn. 1 has been written in an orthogonal local basis, $\phi_{i \alpha}$, defined by the Lowdin's transformation $\phi_{i \alpha}=\sum_{j \beta}\left(S^{-1 / 2}\right)_{i \alpha, j \beta} \psi_{j \beta}, \psi_{j \beta}$ being the local basis used in the DFT-LDA calculation from which we obtain Hamiltonian 1, as explained below $\left(S_{i \alpha, j \beta}\right.$ is the overlap between orbitals $\psi_{i \alpha}$ and $\psi_{j \beta}$ ).

The LD-solution of Hamiltonian 1 is obtained by introducing the kinetic and many-body energies of the system as a function of the orbital occupancies, $n_{i \alpha \sigma}$ [16]. This implies that the total energy is a function of those numbers, $n_{i \alpha \sigma}$, that play the role of the electron density, $\rho(\bar{r})$, in the conventional DFT-approach. Then, we can write the following eqn.:

$$
E\left[\left\{n_{i \alpha \sigma}\right\}\right]=T\left[\left\{n_{i \alpha \sigma}\right\}\right]+E^{H}\left[\left\{n_{i \alpha \sigma}\right\}\right]+E^{X C}\left[\left\{n_{i \alpha \sigma}\right\}\right],
$$

where $T=\left\langle\Psi_{0}\left|\hat{H}^{O E}\right| \Psi_{0}\right\rangle, \Psi_{0}$ being the ground state of the total LD Hamiltonian; $E^{H}$ is the hartree energy and $E^{X C}$ is the exchange-correlation energy associated with Hamiltonian 11. On the other hand [16]:

$$
\begin{aligned}
E^{X}\left[\left\{n_{i \alpha \sigma}\right\}\right]= & -\frac{1}{2} \sum_{i, \alpha \sigma \neq \beta \sigma} J_{i}^{x} n_{i \alpha \sigma} n_{i \beta \sigma} \\
& -\frac{1}{2} \sum_{i \alpha \sigma} J_{i} n_{i \alpha \sigma}\left(1-n_{i \alpha \sigma}\right),
\end{aligned}
$$

an eqn. that yields the exchange energy as the sum of an intrasite contribution and of the intersite interaction between the electron charge, $n_{i \alpha \sigma}$, and its hole, $\left(1-n_{i \alpha \sigma}\right)$. In this eqn., $J_{i}$ is practically the coulomb interaction between charges located in n.n. atoms. Because of the crystal symmetry , we assume that no exchange hole appears in the same atom where the electron is located. On the other hand, we have also shown 16] that the correlation energy is given by:

$$
E^{C}\left[\left\{n_{i \alpha \sigma}\right\}\right]=-\frac{1}{2} \sum_{i \alpha \sigma} f_{i}\left(U_{i}-J_{i}\right) n_{i \alpha \sigma}\left(1-n_{i \alpha \sigma}\right)
$$

where $\left(U_{i}-J_{i}\right)$ is an effective intrasite coulomb interaction between $i$-site orbitals, and $f_{i}$ a quantity ranging between 0 and 1 depending on the importance of the intrasite correlation effects $\left(f_{i}\right.$ is 1 for large values of $\left(U_{i}-J_{i}\right)$ ). Eqns. 2, 3 and 4 allow us to substitute Hamiltonian 1 for an effective Hamiltonian where, instead of the manybody terms, we introduce the local potentials $\left(V_{i \alpha \sigma}^{H}\right.$ and $\left.V_{i \alpha \sigma}^{X C}\right)$ given by:

$$
\begin{aligned}
V_{i \alpha \sigma}^{H} & =\frac{\partial E^{H}\left[\left\{n_{i \alpha \sigma}\right\}\right]}{\partial n_{i \alpha \sigma}}= \\
& =\sum_{\beta \sigma^{\prime} \neq \alpha \sigma} U_{i} n_{i \beta \sigma^{\prime}}+\sum_{j \beta \sigma^{\prime}(j \neq i)} J_{i \alpha, j \beta} n_{j \beta \sigma^{\prime}}
\end{aligned}
$$

$$
\begin{aligned}
V_{i \alpha \sigma}^{X C}= & \frac{\partial E^{X C}\left[\left\{n_{i \alpha \sigma}\right\}\right]}{\partial n_{i \alpha \sigma}}=-\sum_{\beta \neq \alpha} J_{i}^{x} n_{i \beta \sigma} \\
& -J_{i}\left(\frac{1}{2}-n_{i \alpha \sigma}\right)-f_{i}\left(U_{i}-J_{i}\right)\left(\frac{1}{2}-n_{i \alpha \sigma}\right),
\end{aligned}
$$

where $f_{i}$ has been assumed to be constant.

This is the main result of our LD-analysis and shows how to reduce the generalized Hubbard Hamiltonian, eqn. 1, to an effective one-electron Hamiltonian, taking into account all the many-body contributions. Conversely, we can use this equivalence to go from a LDsolution to a generalized Hubbard Hamiltonian: Assume we solve the conventional DFT-LDA eqns for a given crystal (say, paramagnetic $\mathrm{Fe}$ ) using a local orbital basis (as done in Fireball [17] or Siesta [18] codes); then, we can substract from the one-electron levels associated with the orthogonalized orbitals, $\phi_{i \alpha}$, the potentials given by eqns. 5 and 6 . This difference defines Hamiltonian $H^{O E}$ in eqn. 1, and allows us to introduce the Hubbard Hamiltonian by means of the interactions $U, J$ and $J^{x}$. Notice that in this approach we have to calculate these interactions using the orthogonalized orbitals, $\phi_{i \alpha}$. In our actual calculations, we have employed the Fireball code for paramagnetic $\mathrm{Fe}$ and used the corresponding local orbital basis.

Ferromagnetic $\mathrm{Fe}$ has been analyzed in our LDapproach by looking for a magnetic solution where some charge is transferred between spins up and down. This implies that selfconsistent potentials, $V_{i \alpha \uparrow}$ and $V_{i \alpha \downarrow}$, should appear for different spins, in such a way that:

$$
\begin{aligned}
V_{i \alpha \uparrow}= & U_{i}\left(N_{i}-n_{i \alpha \uparrow}\right)+\sum_{j \beta \sigma^{\prime}(j \neq i)} J_{i \alpha, j \beta} n_{j \beta \sigma^{\prime}} \\
& -J_{i}^{x}\left(N_{i \uparrow}-n_{i \alpha \uparrow}\right)-J_{i}\left(\frac{1}{2}-n_{i \alpha \uparrow}\right) \\
& -f_{i}\left(U_{i}-J_{i}\right)\left(\frac{1}{2}-n_{i \alpha \uparrow}\right)
\end{aligned}
$$

where $N_{i}$ is the total charge in the $d$-orbitals for the $i$-site, while $N_{i \uparrow}$ represents the total spin-up charge. Notice how the terms contributing to $V_{i \alpha \uparrow}$ correspond to the Hartree, the intraatomic exchange, the extraatomic exchange and the correlation contributions, respectively. Due to the magnetic polarization, we find changes in the many-body potential w.r.t. the paramagnetic solution. This yields:

$\delta V_{i \alpha \uparrow}=-\left(1-f_{i}\right)\left(U_{i}-J_{i}\right) \delta n_{i \alpha \uparrow}-J_{i}^{x}\left(\delta N_{i \uparrow}-\delta n_{i \alpha \uparrow}\right)$

where the total charge, $N_{i}$, has been assumed to be constant and independent from the atomic magnetization. Eqn. 8 defines how the $i \alpha \uparrow$-level depends on the atomic polarization, $\delta n_{i \alpha \uparrow}$. This quantity should be obtained selfconsistently by means of a band-structure calculation whereby the atomic charges, $\delta n_{i \alpha \uparrow}$, are a function of $\delta V_{i \alpha \uparrow}$. These two conditions yield $\delta n_{i \alpha \uparrow}$ and the crystal magnetization. Eqn. 8 allows us, however, to calcu- 
late directly the Stoner parameter, $I$, which we define as $\left|\delta V_{i \alpha \uparrow} / \delta n_{i \alpha \uparrow}\right|$. Eqn. 8 yields the following result:

$$
I=\left(1-f_{i}\right)\left(U_{i}-J_{i}\right)+4 J_{i}^{x}
$$

assuming $\delta N_{i \uparrow}=5 \delta n_{i \alpha \uparrow}$, as corresponds to $d$ orbitals. In our calculations for $\mathrm{Fe}$, we find $U_{i}=14.7 \mathrm{eV}$ (taking into account atomic relaxation), $J_{i}=6 \mathrm{eV}$ and $J_{i}^{x}=0.83 \mathrm{eV}$.

A word of caution should be introduced here, because in our discussion we have neglected an effect that leads to a further reduction in the effective interaction between orbitals. This is associated with the $s p$-band screening that has been shown by other authors 7 to reduce $\left(U_{i}-J_{i}\right)=U_{i}^{\text {eff }}$ to values close to $5 \mathrm{eV}$. In our calculations, performed introducing a Lindhard dielectric function, we have found that $U_{i}^{\text {eff }}$ is reduced to $4.0 \mathrm{eV}$. If we introduce this value in eqn. 9 and take $f_{i}=0.83$ (the value that corresponds to this reduced interaction), we find $I=4.0 \mathrm{eV}$, in good agreement with DFT-LDA calculations. We have also analyzed how $I$ depends on $U_{i}^{\text {eff }}$ by calculating $f_{i}$ for different intrasite coulomb interactions. Our results show that, in the $2-5 \mathrm{eV}$ range, $\left(1-f_{i}\right) U_{i}^{\text {eff }}$ is almost insensitive to the values of $U_{i}^{\text {eff }}$. These results show that the Stoner parameter is mainly controlled by $J_{i}^{x}$; in other words, ferromagnetism in Fe is an effect mainly associated with the intraatomic first Hund's rule.

Next, we calculate many-body effects introducing a local selfenergy, $\Sigma_{i \alpha \sigma}(\omega)$, within the DMF-approximation. This is a reasonable approximation considering that correlation effects in $\mathrm{Fe}$ are associated with the intrasite coulomb interaction between $d$ orbitals. As discussed in ref [16], $\Sigma_{i \alpha \sigma}(\omega)$ is calculated by an appropriate interpolation between two limits: (1) first, we calculate the atomic limit, assuming $U_{i}^{\text {eff }}$ much larger than the metal bandwidth;(2) second, we obtain the second order selfenergy, $\Sigma_{i \alpha \sigma}^{(2)}(\omega)$, using as the expansion parameter $U_{i}^{\text {eff }} ;(3)$ finally, we calculate the selfenergy interpolating between these two limits.

We should stress that, in this solution, $\Sigma_{i \alpha \sigma}^{(2)}$ is calculated using the local density of states defined by the LD-solution discussed above. Finally, we replace $V_{i \alpha \sigma}^{c}$, in our effective LD-Hamiltonian, by that selfenergy; then, we use a conventional Green-function formalism to calculate the local density of states. At this point, we should comment that consistency between the LD- and the selfenergy formulations imposes the following Luttinger sum rule: $V_{i \alpha \sigma}^{c}=\Sigma_{i \alpha \sigma}\left(E_{F}\right)$. The factor $f_{i}$ in eqn 4 has been determined from this eqn., this procedure satisfying the Luttinger condition automatically. The price we have to pay is the introduction of a selfconsistent loop in the calculation.

Fig. 1 shows our calculated LD- and many-body DOS for ferromagnetic Fe. The comparison between these two density of states shows that correlation effects are important for Fe: first, we notice that the energy difference between the two maxima appearing in the spin-up and spin-down DOS for the LD-solution is reduced by almost a factor of two in the many-body case. This is a typical band narrowing effect appearing around $E_{F}$ and associated with a highly correlated electron gas. On the other hand, we also find that the DOS-structure at energies far away from $E_{F}$ is strongly modified by the many-body solution: in particular, a new satellite structure appears around $5 \mathrm{eV}$ below $E_{F}$. We analyze more in detail these many-body effects by considering the DOS for a particular $\bar{k}$-vector: we have chosen the $P$-point, a case for which there are high quality photoemission data taken along the (111) direction 11, 12].
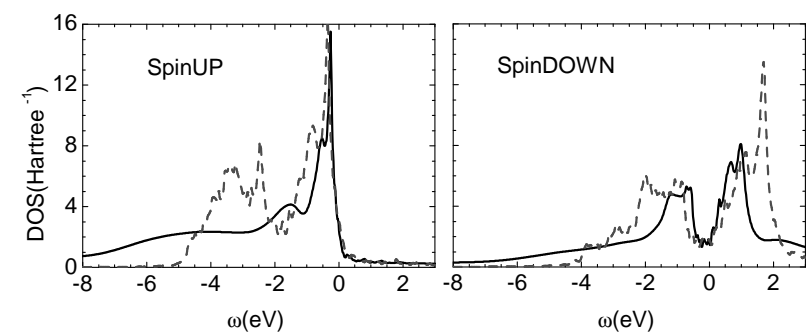

FIG. 1. Total Fe spin DOS for the many-body solution with $U^{\text {eff }}=4 \mathrm{eV}$ (continuous line) and for the DFT-LDA solution (dashed line). Notice the shift of the peaks towards $E_{F}$ when correlation effects are introduced.

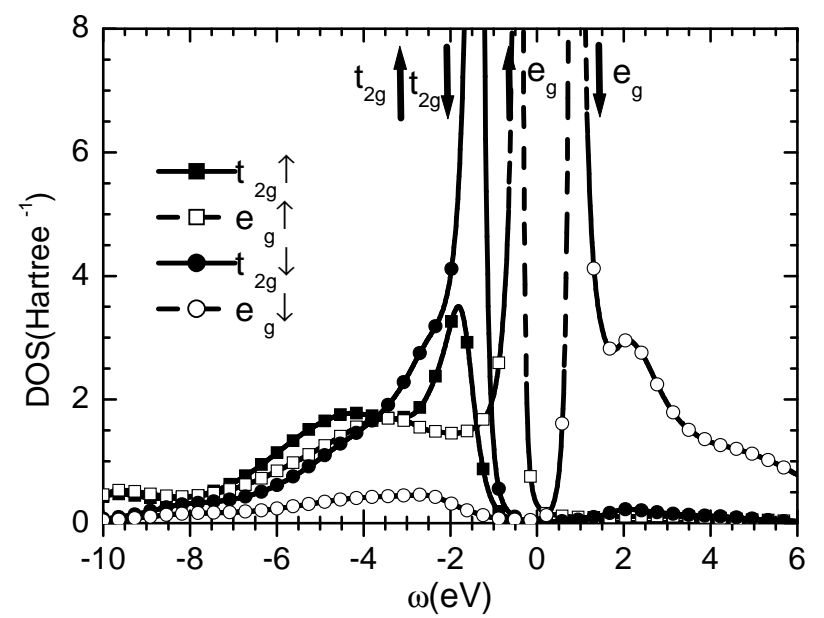

FIG. 2. Spin DOS, split into the $t_{2 g}$ and $e_{g}$ components, at the $P$-point for Fe. The position of the DFT-LDA eigenvalues is indicated by arrows. Notice that the $\mathrm{t}_{2 g}(\mathrm{up})$ state (black squares) has lost its quasiparticle character.

Fig. 2 shows the electron DOS for this $\bar{k}$-vector split into the $t_{2 g}$ and the $e_{g}$-components, and the energy levels corresponding to the DFT-LDA-solution for this $\bar{k}$-point. In this figure we also find the effects already discussed for fig. 1: the $e_{g}(\mathrm{up})$ and $t_{2 g}$ (down) levels located 0.6 $\mathrm{eV}$ and $2.1 \mathrm{eV}$ below $E_{F}$ are shifted to around 0.4 and $1.4 \mathrm{eV}$, respectively. In both cases, we also find some satellites at higher binding energies due to many-body effects. The other $t_{2 g}$ (up)-level located $3.1 \mathrm{eV}$ below $E_{F}$ is completely smeared out by the selfenergy, giving rise to two features: one is almost coinciding in energy with the $t_{2 g}$ (down)-peak, the other one is a very broad peak 
located around $4.5 \mathrm{eV}$ below $E_{F}$. For energies above $E_{F}$, we find the $e_{g}$ (down)-level, located $1.4 \mathrm{eV}$ above $E_{F}$, shifted by many-body effects to $0.9 \mathrm{eV}$. In fig. 3 we compare these results with photoemission data [1],12], by considering the appropriate weight that each state has in the photoemitted electrons: this is done assuming the final state to be a plane wave; in this way we find that, for the $P$-point, the $t_{2 g}$-levels are reduced in intensity by a factor of 3 w.r.t. the $e_{g}$-levels. Comparing this DOS with the photoemitted spectra, we clearly see that the two peaks below $E_{F}$ (at 0.4 and $1.4 \mathrm{eV}$ ) are related to the $e_{g}$ (up) and $t_{2 g}$ (down) states calculated in LD, while the peak above $E_{F}($ at $0.9 \mathrm{eV})$ corresponds to a $e_{g}$ (down) level: these results are in reasonable agreement with the experimental data of refs. 11,12. More importantly, we find that the very broad peak located around $3-4 \mathrm{eV}$ below $E_{F}$ cannot be related directly to the $t_{2 g}($ up)-states found in $\mathrm{LD}$ around $3.1 \mathrm{eV}$ below $E_{F}$ [11]. On the contrary, our results clearly show that this peak is a satellite structure created by many-body effects and appearing as the result of combining the tail intensities of $e_{g}$ and $t_{2 g}$ states (see fig 2 , and remember the factor $1 / 3$ we have to introduce in the weight of the $t_{2 g}$-states).

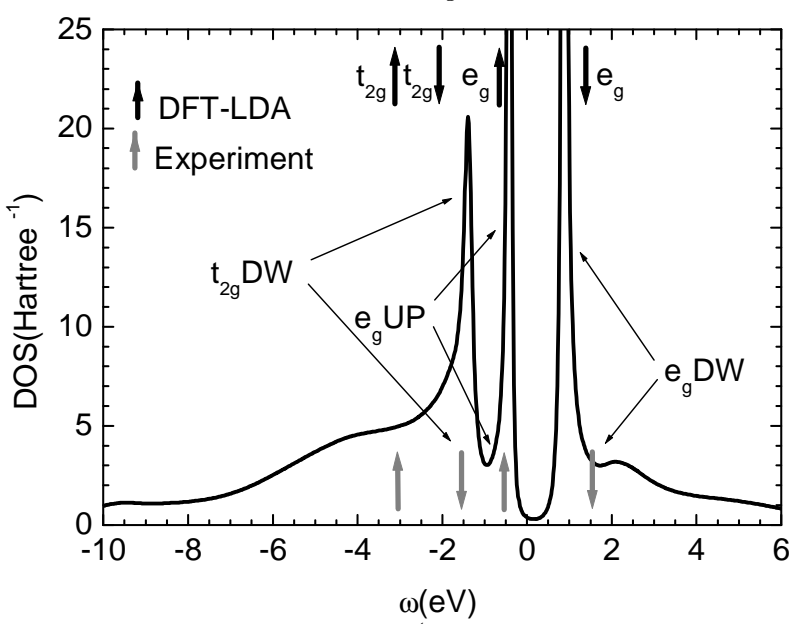

FIG. 3. Quasiparticle spectra (considering the appropriate weight for each state, see text) at the $P$-point for Fe. Black arrows indicate the eigenstates of the DFT-LDA Hamiltonian. Experiments 11, 12] show three well-defined peaks close to $E_{F}$ and a very broad feature around $3 \mathrm{eV}$ below $E_{F}$ traditionally interpreted as the $t_{2 g}$ (up) state (Gray arrows).

This analysis clarifies several contradictory points about the magnetism of iron. Our results show that we can find a reasonable agreement between theory and photoemision data for $U^{\mathrm{eff}}$ as large as $4 \mathrm{eV}$. The reason why a value of $U^{\mathrm{eff}} \simeq 2 \mathrm{eV}$ has been used in the interpretation of these data is the tendency to identify the broad satellite peak, located around $3 \mathrm{eV}$ below $E_{F}$, at the $P$-point, with the quasiparticle level that in DFT-LDA appears around $3.1 \mathrm{eV}$ below $E_{F}$. Our results show, however, that this quasiparticle level has lost its identity due to correlation effects and that it has been modified into a smeared DOS: this new DOS tends to create a satellite structure that should be reinterpreted as due to manybody effects and not as a quasiparticle level reminiscent of the DFT-LDA level. This same effect is also responsible of the satellite peak we find in the total DOS (see fig 1) around $5 \mathrm{eV}$ below $E_{F}$.

In conclusion, we have studied the electronic properties of ferromagnetic Fe using a first-principles LCAOscheme to analyze in detail the role of electron correlations. We find that the correlation potential strongly screens the magnetic effects commonly associated with a local Hubbard interaction: magnetism in $\mathrm{Fe}$ is an effect associated with the first atomic Hund's rule. Moreover, we also find important correlation effects in the spinpolarized DOS. In particular, our analysis shows that the $t_{2 g}(\mathrm{up})$ level located $3.1 \mathrm{eV}$ below $E_{F}$ have lost their quasiparticle identity, due to many-body effects, and tend to create a satellite structure that has been observed experimentally.

This work has been partly funded by the spanish CICYT under contract No. PB-97-0028. F. F. thanks N. H. March for an illuminating discussion.

[1] A.I. Lichtenstein and M.I. Katsnelson, Phys. Rev. B 57, 6884 (1998).

[2] A. Gutierrez and M.F. López, Phys. Rev. B 56, 1111 (1997).

[3] M.I. Katsnelson and A.I. Lichtenstein, J. Phys.: Condens. Matt 11, 1037 (1999).

[4] I. Yang, S.Y. Savrasov and G. Kotliar, Phys. Rev. Lett. 87, 216405 (2001).

[5] See, for instance, H. Ibach and H. Lüth, Solid State Physics (Springer, 1991).

[6] D. A. Papaconstantopoulos, Handbook of the Band Structure of Elemental Solids (Plenum, New York, 1986).

[7] V.I. Anisimov and O. Gunnarson, Phys. Rev. B 43, 7570 (1991).

[8] M.M. Steiner, R.C. Albers and L.J. Sham, Phys. Rev. B 45, 13272 (1992).

[9] N. Majlis, The Quantum Theory of Magnetism (World Scientific, 2000).

[10] A.M. Turner, A.W. Donoho and J.L. Erskine, Phys. Rev. B 29, 2986 (1984).

[11] D.E. Eastman, F.J. Himpsel and J.A. Knapp, Phys. Rev. Lett. 44, 95 (1980).

[12] A. Santoni and F.J. Himpsel, Phys. Rev. B 43, 1305 (1991).

[13] J.C. Stoddart and N. H. March, Annals of Physics 64, 174 (1971).

[14] V.I. Anisimov, F. Aryasetiawan and A.I. Lichtenstein, J. Phys.: Condens. Matter 9, 767 (1997).

[15] A. Georges, G. Kotliar, W. Krauth and M.J. Rozemberg, Rev. Mod. Phys. 68, 13 (1996).

[16] P. Pou et al., Phys. Rev. B 62, 4309 (2000).

[17] A. A. Demkov J. Ortega, O.F. Sankey and M.P. Grumbach, Phys. Rev. B 52, 1618 (1995); J.P. Lewis et al., Phys. Rev. B 64, 195103 (2001).

[18] D. Sánchez-Portal, P. Ordejón, E. Artacho and J.M. Soler, Int. J. Quantum Chem. 65, 453 (1997). 